\title{
Mother-infant interaction in women with depression in pregnancy and in women with a history of depression: the Psychiatry Research and Motherhood - Depression (PRAM-D) study
}

Rebecca H. Bind, Alessandra Biaggi, Aoife Bairead, Andrea Du Preez, Katie Hazelgrove, Freddie Waites, Susan Conroy, Paola Dazzan, Sarah Osborne, Susan Pawlby, Vaheshta Sethna and Carmine M. Pariante

\section{Background}

Little is known about the effects of depression before birth on the quality of the mother-infant interaction.

\section{Aims \\ To understand whether depression, either in pregnancy or in lifetime before pregnancy, disrupts postnatal mother-infant interactions.}

\section{Method}

We recruited 131 pregnant women (51 healthy, 52 with major depressive disorder (MDD) in pregnancy, 28 with a history of MDD but healthy pregnancy), at 25 weeks' gestation. MDD was confirmed with the Structured Clinical Interview for DSM-IV Disorders. Neonatal behaviour was assessed at 6 days with the Neonatal Behavioural Assessment Scale, and mother-infant interaction was assessed at 8 weeks and 12 months with the crittenden CARE-Index.

\section{Results}

At 8 weeks and 12 months, dyads in the depression and historyonly groups displayed a reduced quality of interaction compared with healthy dyads. Specifically, at 8 weeks, $62 \%$ in the depression group and $56 \%$ in the history-only group scored in the lowest category of dyadic synchrony (suggesting therapeutic interventions are needed), compared with $37 \%$ in the healthy group $(P=0.041) ; 48 \%$ and $32 \%$, respectively, scored the same at
12 months, compared with $14 \%$ in the healthy group $(P=0.003)$. At 6 days, neonates in the depression and history-only groups exhibited decreased social-interactive behaviour, which, together with maternal socioeconomic difficulties, was also predictive of interaction quality, whereas postnatal depression was not.

\section{Conclusions}

Both antenatal depression and a lifetime history of depression are associated with a decreased quality of mother-infant interaction, irrespective of postnatal depression. Clinicians should be aware of this, as pregnancy provides an opportunity for identification and intervention to support the developing relationship.

\section{Keywords}

Perinatal psychiatry; depressive disorders; developmental disorders; psychosocial interventions; childhood experience.

\section{Copyright and usage}

(c) The Author(s), 2021. Published by Cambridge University Press on behalf of the Royal College of Psychiatrists. This is an Open Access article, distributed under the terms of the Creative commons Attribution licence (http://creativecommons.org/ licenses/by/4.0/), which permits unrestricted re-use, distribution, and reproduction in any medium, provided the original work is properly cited.
Depression throughout the perinatal period is critical in determining risk to offspring mental health outcomes, ${ }^{1}$ and a disrupted mother-infant interaction is a possible mechanism underpinning this transmission. ${ }^{2}$ Most research has focused on postnatal depression (PND), which can manifest as unresponsiveness to, and withdrawal from the infant, or as intrusive and controlling behaviour, ${ }^{3,4}$ with concomitant reduced sensitivity. ${ }^{2,5}$ Less well-studied, however, is whether depression during the antenatal period - affecting up to $20 \%$ of pregnancies $^{6}$ - can also interfere with the quality of the mother-infant interaction. Indeed, studies have shown that the association between PND and a less optimal mother-infant interaction may be attributable to a continuation of impaired foetal attachment from pregnancy into the postnatal period, ${ }^{7}$ and that antenatal depressive symptoms are associated with poorer bonding and increased maternal unresponsiveness postnatally. ${ }^{8-10}$ However, these studies have used self-report measures of mood symptoms, largely in community samples. To our knowledge, no prior studies have looked at mother-infant interactions in the context of clinically significant major depressive disorder (MDD), confirmed through a structured, standardised diagnostic interview, in pregnant women recruited from perinatal mental health services It is thus important to fill this gap, as antenatal depression at a clinical level may have a more severe effect on the dyadic interaction.
Of note, there is also little evidence on whether a lifetime history of MDD alone can affect mother-infant interactions, in the absence of depression in pregnancy. Only one study, to our knowledge, has examined this, and found that mothers (and fathers) with a history of depression were more likely to display negative affect when interacting with their infants, even if they were well in the perinatal period. ${ }^{11}$ Given that a history of MDD is one of the largest risk factors for perinatal MDD, ${ }^{6}$ and previous literature finds associations between perinatal MDD and difficulties in the interaction, it is worth investigating whether these difficulties may be present even in women with a past history of depression who do not necessarily meet clinical criteria for a depressive episode during pregnancy, either because of subsyndromal symptom carryover into pregnancy, or because a past history may interfere with biological mechanisms underpinning the mother-infant bond. ${ }^{12,13}$

Finally, it is important to establish whether any putative effects of maternal depression on the mother-infant interaction are confounded by other maternal and infant variables that are relevant to the mother-infant interaction, such as maternal childhood maltreatment and socioeconomic stress, which not only predispose women to later depression, ${ }^{14,15}$ but are also associated with decreased maternal sensitivity; ${ }^{16,17}$ or PND, which is associated with both depression in pregnancy and a history of depression, ${ }^{6}$ 
and is thought to affect maternal sensitivity; ${ }^{5}$ or suboptimal neonatal behaviour, which has been associated with both antenatal depression ${ }^{18}$ and decreased dyadic synchrony. ${ }^{19}$ Of note, although prior studies have assessed the effect of maternal depression on separate maternal and infant domains of behaviour, the impact on dyadic synchrony - a precursor to infant attachment that reflects behavioural patterns of both members of the dyad together as one unit - has not been previously studied.

\section{Current study}

Thus, the novelty of our objectives are as follows: we examined maternal depression before birth (either during pregnancy or lifetime before pregnancy); we studied mother-infant interaction, both through dyadic synchrony and through separate maternal and infant domains of behaviour; we assessed the interaction at two time points ( 8 weeks and 12 months) in the first postnatal year; and we tested whether women with depression presented with clinical or sociodemographic risk factors that may have contributed to the putative association between maternal depression and difficulties in the mother-infant interaction.

We overall hypothesized that (a) MDD experienced during or before pregnancy would be associated with decreased dyadic synchrony across the postnatal period and (b) based on previous literature above, possible confounders of this relationship may be maternal history of childhood maltreatment, maternal socioeconomic difficulties, maternal PND and suboptimal infant neonatal behaviour.

\section{Method}

\section{Design}

The present study is part of the Psychiatry Research and Motherhood - Depression (PRAM-D) study, ${ }^{18}$ a prospective longitudinal study of women in pregnancy and the postpartum, and their offspring. The sample included 131 women: 51 healthy women, attending their routine antenatal ultrasound scan at King's College Hospital [healthy group]; 52 women diagnosed with depression, referred to the Maudsley Perinatal Psychiatry Services [depression group]; and 28 'history-only' women, who had a history of MDD but no current diagnosis, recruited from either their regular antenatal scan or the psychiatric clinical service (where they were referred for assessment only, because of the historical vulnerability) [history-only group]. Women and their offspring were assessed from 25 weeks' gestation (baseline) until 1 year postnatal.

The authors assert that all procedures contributing to this work comply with the ethical standards of the relevant national and institutional committees on human experimentation and with the Helsinki Declaration of 1975, as revised in 2008. All procedures involving human patients were approved by the King's College Hospital Research Ethics Committee (approval number REC 07/ Q0703/48). All participants provided written informed consent.

\section{Participants and procedure}

Inclusion criteria for the study were as follows: women of at least 18 years with a singleton pregnancy; for the depression group, a DSMIV diagnosis of MDD in the current pregnancy, at or before 25 weeks' gestation, as maternal responsiveness is thought to solidify by then; ${ }^{20}$ for the history-only group, a history of MDD but no diagnosis of MDD throughout the entire pregnancy (if women developed depression in pregnancy after 25 weeks', they were excluded from analyses); and, for healthy women, no current or past DSM-
IV diagnoses. Exclusion criteria were as follows: uterine anomaly, obstetric complications, severe or relevant chronic medical conditions, history of psychosis or bipolar affective disorder and antidepressant usage at baseline (but not before or after baseline).

Of the 131 women assessed at baseline for sociodemographic and clinical information, 130 dyads were seen at 6 days postnatal to assess neonatal behaviour, 121 dyads were seen at 8 weeks postnatal and 112 dyads were seen at 12 months postnatal, to assess sociodemographic and clinical variables, as well as the mother-infant interaction (see study flow chart in Fig. 1). There were no significant differences in attrition rates between the three groups (see Supplementary Table 1 available at https:// doi.org/10.1192/bjo.2021.52), nor in socioeconomic variables or clinical variables between participants who remained in the study and those who dropped out (see Supplementary Table 2).

\section{Sociodemographic and clinical measures}

Sociodemographic and socioeconomic (SES) variables were ascertained with a semi-structured interview. As SES variables were very highly correlated with each other, a composite SES score was created to explore the possibility of cumulative risk, as has been previously done. ${ }^{21}$ It encompassed maternal age, ethnicity (white versus ethnic minority), marital status (married or cohabiting versus single with or without partner), occupation (employed or student versus non-employed or full-time mother) and qualification level (GCSE or lower versus A-level or higher), all assessed at baseline, whereby a score of 0 represented the mean status across the sample, a negative score represented more risk factors for adversity and a positive score represented more protective factors. These particular SES variables were chosen as young age, belonging to an ethnic minority group, being single, being unemployed and having lower education qualifications have all been previously associated with maternal depression. ${ }^{6}$

\section{Maternal history of childhood maltreatment}

Measures of maternal history of sexual, physical and emotional abuse, and neglect were obtained with the cut-off $\mathrm{B}$ of the Childhood Experience of Care and Abuse Questionnaire (CECA-Q), ${ }^{22}$ which has been used extensively in this and other cohorts. ${ }^{23}$ Outcomes were based on experiences that occurred in childhood from age 0 to 17 years. Presence of childhood maltreatment was rated based on severity and frequency, according to guidelines published by Bifulco et al. ${ }^{22}$ Specifically, the CECA-Q contains two different cut-offs (A and B) for each of the different types of maltreatment, derived from validation against the interview measure. ${ }^{22}$ Cut-off $B$ has been suggested as the optimal one to use for adulthood disorders, and thus was appropriate for our study. Cut-off points for cut-off B of each maltreatment type are described below.

Physical abuse. Participants who responded yes to the screening question were asked follow-up questions, including how often this abuse occurred and if they were injured as a result. Experience of physical abuse was rated separately for each parent figure, and a score was generated for each parental figure, ranging from 0 to 4 . Scores of 0-2 were then recoded as 0 (minimal physical abuse); scores of 3 or 4 were recoded as 1 (severe physical abuse).

Sexual abuse. If participants answered yes, a total score for the first unwanted sexual experience was created by summing responses, and each unwanted sexual experience was rated $0-5$; furthermore, scores of 0 or 1 were recoded as 0 (minimal sexual abuse) and scores of $2-5$ were recoded as 1 (severe sexual abuse). 


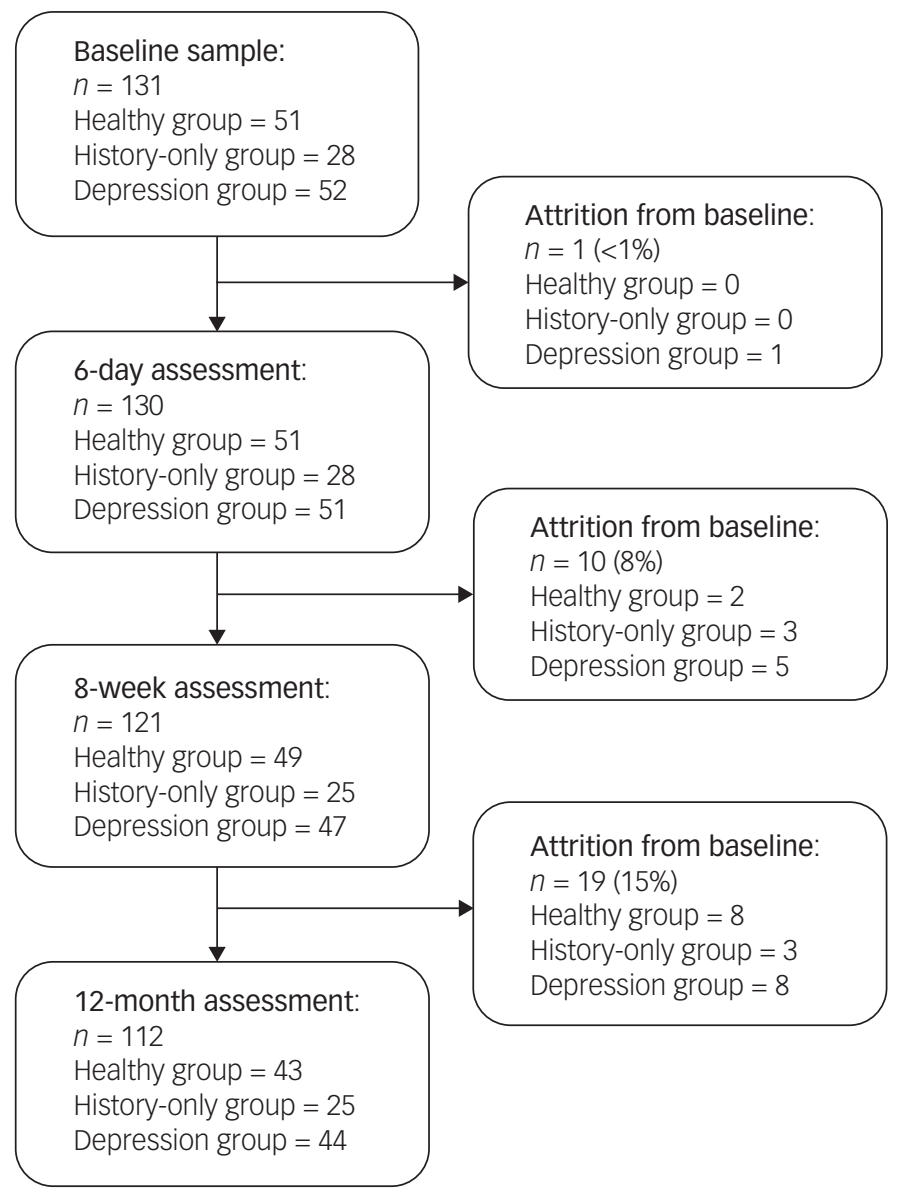

Fig. 1 Psychiatry Research and Motherhood - Depression (PRAM-D) study participant flow chart.

Antipathy and neglect. Antipathy and neglect were assessed with 16 self-report items about either parent in childhood. Each item was rated on a five-point Likert scale from 1 (yes definitely), to 5 (no, not at all). Eight items assessed antipathy and the other eight items measured neglect, with scores ranging from 8 to 40 . For antipathy, scores of 8-27 were recoded as 0 , no antipathy; scores of $\geq 28$ were recoded as 1 , severe antipathy. For parental neglect, scores of 8-24 were recoded as 0 , no neglect; scores of $\geq 25$ were recoded as 1 , severe neglect.

\section{Diagnosis of depression}

Past and current Axis I diagnoses were assessed at baseline, using the Structured Clinical Interview for DSM-IV Disorders (SCID-I), ${ }^{24}$ for which a diagnosis of MDD was given if the clinical criteria for depression were met. Participants were readministered the SCID-I at 8 weeks and 12 months postnatal, to assess for episodes of PND since the previous assessment. Additionally, self-report questionnaires (Beck Depression Inventory (BDI) ${ }^{25}$ and State-Trait Anxiety Inventory $(\mathrm{STAI})^{26}$ ) were collected to assess current depressive and anxious symptoms.

\section{Neonatal behaviour}

Neonatal behaviour was evaluated at 6 days postnatal, using the Neonatal Behavioural Assessment Scale (NBAS). ${ }^{27}$ The NBAS assesses neurobehavioural function in neonates up to 2 months of age, and produces information based on 28 behavioural items and 18 reflex items. These items are then divided into five clusters of functioning, covering key developmental areas, and include regulation of the autonomic system, motor system, regulation of state, range of state and orientation (the infant's ability to attend to visual and auditory stimuli, as well as their overall quality of alertness; also referred to as social-interactive abilities). For the purposes of this study, we only include the orientation cluster of the NBAS, as it assesses an infant's quality of alertness, is indicative of socialinteractive abilities with the examiner and has previously been associated with dyadic interaction. ${ }^{28}$ Data for the healthy women and women with depression in an overlapping sample have been published before, ${ }^{18}$ but we present new data from the history-only group (see Results and Table 1) and its association with motherinfant interaction

\section{Mother-infant interaction assessment}

The dyadic relationship was assessed with the Crittenden ChildAdult Relationship Experimental Index (CARE-Index), ${ }^{29}$ a reliable and valid coding method used both clinically and in research with infants up to 15 months of age. We filmed 3-min interactions at 8 weeks and 12 months postnatal, for which mothers were instructed to play with their babies as they normally would. Quality of the interaction was rated across three scales: maternal behaviour, infant behaviour and dyadic synchrony. Mothers and infants were given scores based on seven aspects of behaviour: facial expression, vocal expression, position and body contact, affection and arousal, turn-taking contingencies, control and choice of activity.

The CARE-Index classes maternal behaviour into the following patterns: sensitivity, control and unresponsiveness. Sensitivity is defined as any pattern of behaviour that 'pleases the infant and increases the infant's comfort and attentiveness and reduces its distress and disengagement'; ${ }^{30}$ the two inverse behaviours of sensitivity 
are control, indicating intrusiveness or hostility, and unresponsiveness, indicating withdrawal from/unconnectedness to the baby. Likewise, infant behaviour is classed into cooperativeness (being pleasantly connected to the mother) and three inverse behaviours: compulsiveness, presenting as fear or compliance; difficultness, presenting as negative connectedness and over-arousal; and passiveness, presenting as withdrawn and affectless behaviour. Both members can express behaviours across all patterns, but will typically display a dominant pattern. Patterns were scored from 0 to 14 , where a higher score reflected greater presence of that behaviour.

Finally, dyadic synchrony is a global indication of how in-tune the dyad is, and how well they are interacting; that is, the overall quality of the interaction. It is rated $0-14$, and divided into four categories, with suggested corresponding interventions when used clinically: risk ( $0-4$, 'need for parental psychotherapy and possible parent-child separation'); inept (5-6, 'voluntary parent-infant intervention'); adequate (7-10, 'optional parent education, but no intervention necessary') and sensitive (11-14, 'no intervention necessary'). As dyadic synchrony is highly correlated with maternal sensitivity and infant cooperativeness, the present study only reports on dyadic synchrony. Additionally, as behavioural patterns are scored proportionally to each other, the present study only includes mothers' unresponsiveness (negatively correlated with control) and infants' compulsivity and passiveness (negatively correlated with difficultness).

The primary trained rater (R.H.B.) has achieved level II reliability with the CARE-Index course (mean correlation coefficient of 0.85 across all scales); reliability was also established between trained raters (blind to maternal mental health status), with an interclass correlation coefficient of 0.90 for dyadic synchrony at 8 weeks and 0.95 at 12 months.

\section{Statistical analysis}

Analyses were conducted with SPSS Statistics version 24 for MacOS (IBM, UK). Before analyses, data were checked for missing data, outliers, accuracy and normality. Main assumptions of normality and homoscedasticity were tested to ensure representativeness of our sample. If violated, data were either log-transformed or non-parametric analyses were conducted. Pearson's $\chi^{2}$ was used for categorical data, including sociodemographic and clinical variables, and the $z$-test was used to compare the three groups. ANOVA was used to compare means for sociodemographic and clinical variables. Kruskal-Wallis test was used to compare means for the mother-infant interaction, with Bonferroni corrections for post hoc comparisons between each of the groups. Analysis of covariance was used for covariate analyses of the interaction. Finally, hierarchical linear regressions were used for prediction modelling for dyadic synchrony. Means (s.e.) are presented in graphs.

\section{Results}

\section{Sample characteristics}

Antenatal risk factors

Antenatal characteristics are presented in Table 1. In terms of antenatal clinical symptoms, women in the depression group, by definition, reported higher symptoms of depression and anxiety on the BDI and STAI $(P<0.001$ for all comparisons); moreover, women in the history-only group displayed more anxious symptoms on the STAI than women in the healthy group $(P=0.013)$. Furthermore, similar proportions in both the depression and history-only groups took antidepressants in pregnancy $(37 \%$ and $21 \%$, respectively) before or after the baseline assessment. With regard to past depression, women in the depression and historyonly groups were equally likely to have a history of recurrent depression before pregnancy, defined as two or more prior episodes (58\% and $63 \%$, respectively).

Table 1 also presents the aforementioned SES and clinical risk factors for antenatal and/or postnatal depression in the three groups. ${ }^{6}$ Women in both the depression and history-only groups had similar rates of exposure to childhood maltreatment, both of which were higher than women in the healthy group $(63 \%$ and $52 \%$ v. $16 \%$, respectively; $P<0.001)$. However, only women in the depression group were also more likely to have lower education qualifications (versus women in the healthy group), be single (versus women in the history-only and healthy groups), be unemployed (versus women in the healthy group), belong to an ethnic minority group (versus women in the history-only group) and, above all, have a lower composite SES score (versus women in the healthy group); that is, a score encompassing age, ethnicity, marital status, occupation and qualification level at baseline $(P=0.001-0.04$; see Table 1$)$. Because of the group differences in history of childhood maltreatment and SES score, they were included in subsequent univariate correlations and hierarchical regression models predicting dyadic synchrony (see Tables 2 and 3).

\section{Postnatal outcomes}

Postnatal characteristics are also presented in Table 1. There were no statistically significant group differences in mode of delivery, infant sex, feeding method or problems, or total duration of breastfeeding.

We evaluated the prevalence of PND by using the SCID-I across three time points: birth to 8 weeks, 8 weeks to 12 months and birth through 12 months. Overall, women in both the depression and history-only groups were more likely to experience PND in the 12 -month postnatal period than women in the healthy group (44\% and $36 \% v$. $7 \%$, respectively; $P<0.002$ ). Between birth and 8 weeks, women in the depression group had the highest rates of PND, followed by women in the history-only and healthy groups ( $21 \%$ v. $11 \%$ v. $2 \%$, respectively; $P=0.009$ ), but between 8 weeks and 12 months, women in the depression and history-only groups had similar rates $(38 \%$ and $32 \%$ v. $4 \%$, respectively; $P<0.01)$. As this variable differed between the groups, it was included in subsequent univariate correlations and hierarchical regression models predicting dyadic synchrony (see Tables 2 and 3 ).

Finally, NBAS orientation abilities (the ability for the infant to attend to auditory and visual stimuli at 6 days) was significantly poorer in infants of women in both the depression and historyonly groups than in infants of women in the healthy group (6.3 \pm 1.5 and $6.4 \pm 1.7$ v. $7.7 \pm 0.9$, respectively; $F(2,127)=14.6 ; P<$ 0.001; Tukey post hoc test $P<0.001$ for both comparisons versus healthy group; $P=0.94$ between depression and history-only groups). As this variable differed between the groups, it was included in subsequent univariate correlations and hierarchical regression models predicting dyadic synchrony (see Tables 2 and 3).

\section{Dyads in the depression and history-only groups have less optimal mother-infant interaction at both 8 weeks and 12 months postnatal}

We compared scores on the CARE-Index between dyads in the depression, history-only, and healthy groups, at 8 weeks and 12 months postnatal (see Fig. 2).

Dyads in both the depression and history-only groups had lower dyadic synchrony scores compared with the healthy group, at both 8 weeks $(4.6 \pm 3.5$ and $4.3 \pm 2.9$ v. $5.7 \pm 2.4$, respectively) and 12 months ( $5.6 \pm 3.2$ and $5.8 \pm 2.8$ v. $7.7 \pm 2.9$, respectively), with no difference between the depression and history-only groups $\left(H_{(2)}=9.06\right.$, 


\section{Sample characteristics at baseline}

\section{Age, years, mean (s.d.)}

Ethnicity, white, $n$ (\%)

Education, A level or higher, $n(\%)$

Employment status, employed, $n$ (\%)

Marital status, married or cohabiting $n$ (\%)

SES score, mean (s.d.)

Number of past episodes of MDD, $>2, n$ (\%)

Antidepressant usage in pregnancy, yes,

$n(\%)$

Parity, primiparous, $n$ (\%)

BDI score at baseline, mean (s.d.)

STAl-S score at baseline, mean (s.d.)

Maternal history of childhood maltreatment, yes, $n(\%)$ Sample characteristics in the postnatal period

Mode of delivery, vaginal, $n$ (\%)

infant sex female, $n$ (\%)

NBAS orientation cluster at 6 days,

mean (s.d.)

Infant age in days at 8-week assessment, mean (s.d.)

Feeding method at 8 weeks, breast, $n$ (\%)

Any feeding problems, yes, $n(\%)$

Antidepressant usage at 8 weeks, yes, $n$ (\%)

SCID diagnosis of MDD between birth and 8 weeks, yes, $n(\%)$

$\mathrm{BDI}$ score at 8 weeks, mean ( $\mathrm{s}$. )

STAI-S score at 8 weeks, mean (s.d.)

Infant age in days at 12-month assessment, mean (s.d.)

otal months of breastfeeding at 12 months, mean (s.d.)

Antidepressant usage at 12 months, yes,

SID diagnosis of MDD between 8 weeks and 12 months, yes, $n$

$(\%)$
Any SCID diagnosis of MDD between birth and 12 months, yes, $n(\%)$

BDI score at 12 months, mean (s.d.)

STAI-S score at 12 months, mean (s.d.)

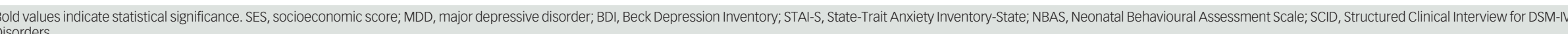

$\begin{array}{cc}\begin{array}{c}\text { Healthy group } \\ (\boldsymbol{n}=\mathbf{4 5 - 5 1 )}\end{array} & \begin{array}{c}\text { History-only group } \\ (\boldsymbol{n}=\mathbf{2 7 - 2 8 )}\end{array} \\ 31.9(4.4) & 32.8(5.9) \\ 40(78.4) & 25(89.3) \\ 47(92.2) & 22(78.6) \\ 45(88.2) & 23(82.1) \\ 45(88.2) & 23(82.1) \\ 0.45(0.7) & 0.24(0.7) \\ - & 17(63.0) \\ - & 6(21.4) \\ 26(51.0) & 17(60.7) \\ 3.6(2.5) & 5.2(3.8) \\ 26.9(6.8) & 34.2(11.1) \\ 7(15.6) & 14(51.9)\end{array}$
$(n=49-51)$

$37(75.5)$

$23(46.9)$

$7.68(0.9)$

History-only group

\section{$(n=24-28)$}

$17(70.8)$

$10(41.7)$

$6.41(1.7)$

$68.69(15.0)$

30 (61.2)

$11(42.3)$

$0(0)$

$1(2.0)$

$3.02(3.0)$
$25.91(7.5)$

81.30 (24.9)

$13(54.2)$

$5(20.5)$

$5(20.8)$

$3(10.7)$

$33.96(9.2)$

$396.90(22.1)$

8.22 (3.9)

$421.80(42.0)$

$6.81(3.9)$
$5(19.2)$

$2(4.3)$

$8(32.0)$

$9(36.0)$

$6.17(5.5)$

$34.10(9.8)$
Statistical test and significance

( $n=47-52)$

$31.0(6.6)$

$33(63.5)$

35 (67.3)

32 (61.5)

$28(53.8)$

$-0.33(1.2)$

$45(57.7)$

19 (36.5)

$23(44.2)$

$19.8(12.9)$

$50.0(13.1)$

$30(62.5)$

$F(2,128)=0.95, P=0.39$

$\chi^{2}(2)=7.02, P=0.030$

$\chi^{2}(2)=9.73, P=0.008$

$\chi^{2}(2)=10.82, P=0.04$

$\chi^{2}(2)=18.27, P=0.001$

$H_{(2)}=11.08, P=0.004$

$\chi^{2}(1)=4.08, P=0.13$

$\chi^{2}(1)=1.93, P=0.16$

$\chi^{2}(2)=6.68, P=0.15$
$F(\mathbf{2}, \mathbf{1 2 0})=\mathbf{5 1 . 4 4}, \boldsymbol{P}<\mathbf{0 . 0 0 1}$

$F(2,120)=59.10, P<0.001$

$\chi^{2}(2)=22.19, P<0.001$

\section{pression gro}

Statistical test and

36 (78.3)

$22(46.8)$

significance

$\chi^{2}(2)=0.63, P=0.96$
$\chi^{2}(2)=0.21, P=0.90$

$79.00(24.2) \quad H_{(2)}=7.26, P=0.027$

$21(44.7) \quad \chi^{2}(2)=3.33, P=0.50$

12 (33.3) $\quad \chi^{2}(2)=0.80, P=0.67$

15 (31.9) $\quad \chi^{2}(2)=17.65, P<0.001$

$11(21.2) \quad \chi^{2}(2)=9.37, P=0.009$

$11.33(9.9) \quad F(2,111)=19.22, P<0.001$

$41.82(13.6) \quad F(2,110)=25.50, P<0.001$

402.80 (27.6) $\quad H_{(2)}=7.97, P=0.019$

$8.01(3.7) \quad F(2,89)=23.45, P=0.45$

$16(34.0) \quad \chi^{2}(2)=\mathbf{1 8 . 2 6}, P<0.001$

$17(37.8) \quad \chi^{2}(2)=15.59, P<0.001$

$20(44.4) \quad \chi^{2}(2)=17.51, P<0.001$

$10.95(11.1) \quad F(2,100)=10.04, P<0.001$

38.99 (12.1) $\quad F(2,100)=12.29, P<0.001$
Post hoc differences

History-only $v$. depression

Healthy $v$. depression

Healthy $v$. depression

Healthy $v$. depression, history-only $v$. depression

Healthy $v$. depression

Healthy $v$. depression, history-only $v$. depression

Healthy $v$. history-only, healthy $v$. depression, history-only $v$. depressed

Healthy $v$. history-only, healthy $v$. depression

\section{Post hoc differences}

Healthy $v$. history-only, healthy $v$. depression

Healthy $v$. history-only, healthy $v$. depression

Healthy $v$. history-only, healthy $v$. depression

Healthy $v$. depression

Healthy $v$. depression, history-only $v$. depression

Healthy $v$. history-only, healthy $v$. depression," history-only $v$. depression

Healthy $v$. history-only, history-only $v$. depression

Healthy $v$. history-only, healthy $v$. depression

Healthy $v$. history-only, healthy $v$. depression

Healthy $v$, history-only, healthy $v$, depression

Healthy $v$. depression, history-only $v$ depression Healthy $v$. history-only, healthy $v$. depression 
Table 2 Correlations between maternal and infant variables with group differences and dyadic synchrony

\begin{tabular}{|c|c|c|}
\hline & $\begin{array}{l}\text { Dyadic } \\
\text { synchrony } \\
\text { at } 8 \text { weeks }\end{array}$ & $\begin{array}{c}\text { Dyadic } \\
\text { synchrony } \\
\text { at } 12 \text { months }\end{array}$ \\
\hline SES score & $0.29 * *$ & $0.38^{* *}$ \\
\hline History of maternal childhood maltreatment ${ }^{b}$ & -0.11 & -0.14 \\
\hline BDI score during pregnancy ${ }^{a}$ & 0.01 & -0.06 \\
\hline STAI-S score during pregnancy ${ }^{a}$ & -0.06 & -0.16 \\
\hline Antidepressants during pregnancy ${ }^{b}$ & -0.17 & -0.17 \\
\hline NBAS orientation score ${ }^{a}$ & $0.20^{*}$ & $0.25^{\star *}$ \\
\hline Postnatal MDD birth to 8 weeks $s^{b}$ & $-0.19 *$ & 0.01 \\
\hline BDI score at 8 weeks ${ }^{a}$ & 0.06 & -0.02 \\
\hline STAI-S score at 8 weeks ${ }^{a}$ & -0.07 & $-0.23^{*}$ \\
\hline Antidepressants at 8 weeks ${ }^{b}$ & -0.06 & -0.09 \\
\hline Postnatal MDD at 8 weeks ${ }^{b}$ & 0.01 & -0.10 \\
\hline $\mathrm{BDI}$ score at 12 months $^{\mathrm{a}}$ & 0.01 & 0.08 \\
\hline STAI-S score at 12 months $^{\mathrm{a}}$ & 0.02 & -0.18 \\
\hline Antidepressants at 12 months ${ }^{b}$ & -0.07 & -0.03 \\
\hline Postnatal MDD 8 weeks to 12 months ${ }^{b}$ & 0.12 & 0.03 \\
\hline \multicolumn{3}{|c|}{$\begin{array}{l}\text { Bold values indicate statistical significance. SES, socioeconomic score; BDI, Beck } \\
\text { Depression Inventory; STAI-S, State-Trait Anxiety Inventory-State; NBAS, Neonatal } \\
\text { Behavioural Assessment Scali; MDD, major depressive disorder. } \\
\text { a. Spearman's correlation coefficients are presented. } \\
\text { b. Point-biserial correlation coefficients are presented. } \\
\star{ }^{\prime} P<0.05,{ }^{*} P<0.01 \text {. }\end{array}$} \\
\hline
\end{tabular}

$P=0.011$ at 8 weeks and $H_{(2)}=12.85, P=0.002$ at 12 months; Bonferroni correction $P=0.026$ (healthy $v$. depression), $P=$ 0.048 (healthy $v$. history-only) and $P=0.002$ (healthy $v$. depression), $P=0.038$ (healthy $v$. history-only), respectively). Notably, at 8 weeks, $62 \%$ of dyads in the depression group and $56 \%$ of dyads in the history-only group scored in the risk category (dyadic synchrony of $0-4$, clinically indicating a need for mother-infant therapeutic interventions ${ }^{30}$ ), compared with $37 \%$ of dyads in the healthy group $\left(\chi^{2}(2)=6.38, P=0.041\right)$; and at 12 months, $48 \%$ of dyads in the depression group and $32 \%$ of dyads in the history-only group continued to score in the risk range, compared with $14 \%$ of dyads in the healthy group $\left(\chi^{2}(2)=11.56, P=0.003\right)$.

In addition, at 12 months, both women in the depression and history-only groups were significantly more unresponsive (higher scores) than women in the healthy group $(4.6 \pm 3.7$ and $4.4 \pm 3.4$ v. $2.5 \pm 2.9$, respectively; $H_{(2)}=9.30 ; P=0.010$; Bonferroni correction $P=0.020$ (healthy $v$. depression) and 0.045 (healthy $v$. history-only), respectively), again with no difference between women in the depression and history-only groups. There were no other differences in patterns of behaviour between groups.

Of note, infants of the depression and history-only groups were significantly older than those of the healthy group at both the 8week and 12-month assessments (see Table 1), because of difficulties in organising visits for these mothers because of their increased vulnerability and complexity; however, we repeated the 8-week and 12 -month analyses on dyadic synchrony with infant age as a covariate, and our results did not change $(F(2,114)=4.47, P=0.014$ at 8 weeks; $F(2,105)=2.43, P=0.010$ at 12 months, respectively).

\section{Variables predicting dyadic synchrony at 8 weeks and 12 months}

To determine whether clinical and sociodemographic variables contributed to the disrupted dyadic synchrony in conjunction with depression, we first identified variables that differed between the three groups (see Table 1) and are hypothesised to have a role in mother-infant interaction, according to previous literature. We then conducted univariate correlations between these variables and dyadic synchrony. As such, variables that were correlated with dyadic synchrony included maternal SES score, infant NBAS orientation score at 6 days, maternal STAI score at 8 weeks and maternal presence of PND between birth and 8 weeks (see Table 2). Contrary to hypotheses, and surprisingly, maternal history of childhood maltreatment was not associated with dyadic synchrony, and was thus excluded from further analyses. Furthermore, as maternal STAI score and the presence of PND were highly correlated with each other $(r=0.41, P<0.001)$, we chose to only include PND in follow-up analyses because of its presence in the literature.

Thus, the final variables included in a hierarchical regression predicting dyadic synchrony at 8 weeks and 12 months were as follows: maternal SES score (encompassing age, ethnicity, qualification, employment and marital status), infant NBAS orientation score and presence of PND between birth and 8 weeks. The hierarchical regression models for dyadic synchrony at 8 weeks and 12 months are presented in Table 3.

At 8 weeks, SES score was significantly associated with dyadic synchrony and accounted for $8 \%$ of the variance; furthermore, NBAS orientation score was also significantly associated with

\begin{tabular}{|c|c|c|c|}
\hline Factors & $R^{2}$ & $\begin{array}{l}\text { Statistical significance } \\
\text { of change in } R^{2}\end{array}$ & Coefficient $(95 \% \mathrm{Cl})$ \\
\hline \multicolumn{4}{|l|}{ Dyadic synchrony at 8 weeks $(n=109)$} \\
\hline SES score & 0.08 & 0.003 & 0.07 (0.03 to 0.12) \\
\hline SES score plus orientation score & 0.13 & 0.020 & 0.06 (0.01 to 0.10$), 0.04$ (0.01 to 0.07 ) \\
\hline SES score plus orientation score plus PND & 0.13 & 0.748 & 0.06 (0.01 to 0.10$), 0.04$ (0.01 to 0.07$), 0.02$ (-0.08 to 0.11$)$ \\
\hline $\begin{array}{l}\text { SES score plus orientation score plus PND plus maternal } \\
\text { group: }\end{array}$ & 0.16 & 0.256 & 0.05 (0.00 to 0.10$), 0.03$ ( -0.01 to 0.06$), 0.04$ ( -0.06 to 0.14 ) \\
\hline Healthy group & & & Reference \\
\hline Depression group & & & $-0.07(-0.19$ to 0.04$)$ \\
\hline History-only group & & & $-0.10(-0.22$ to 0.03$)$ \\
\hline \multicolumn{4}{|l|}{ Dyadic synchrony at 12 months $(n=103)$} \\
\hline SES score & 0.20 & $<0.001$ & $0.10(0.06$ to 0.14$)$ \\
\hline SES score plus orientation score & 0.20 & 0.406 & 0.09 (0.05 to 0.13$), 0.01$ ( -0.02 to 0.04$)$ \\
\hline SES score plus orientation score plus PND & 0.20 & 0.834 & 0.09 (0.05 to 0.13$), 0.01$ ( -0.02 to 0.04$), 0.01$ ( -0.07 to 0.09 ) \\
\hline $\begin{array}{l}\text { SES score plus orientation score plus PND plus maternal } \\
\text { group: }\end{array}$ & 0.23 & 0.248 & 0.09 (0.05 to 0.13$), 0.00$ (-0.03 to 0.03$), 0.03$ ( -0.06 to 0.12 ) \\
\hline Healthy group & & & Reference \\
\hline Depression group & & & $-0.06(-0.16$ to 0.05$)$ \\
\hline History-only group & & & $-0.09(-0.20$ to 0.02$)$ \\
\hline
\end{tabular}




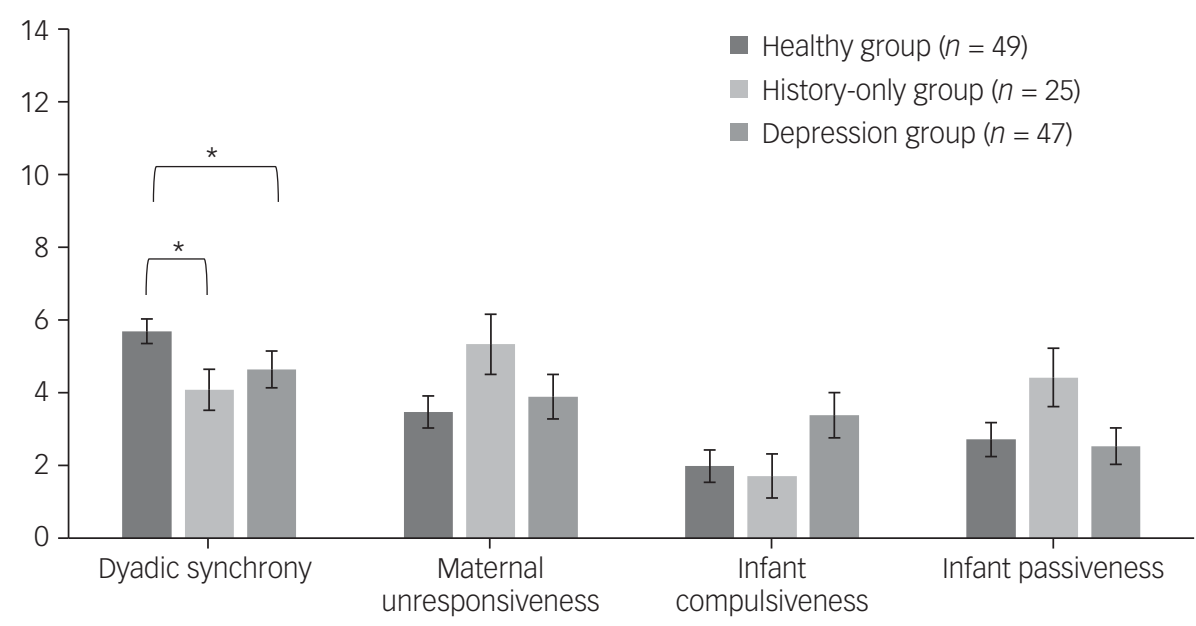

8 weeks postnatal

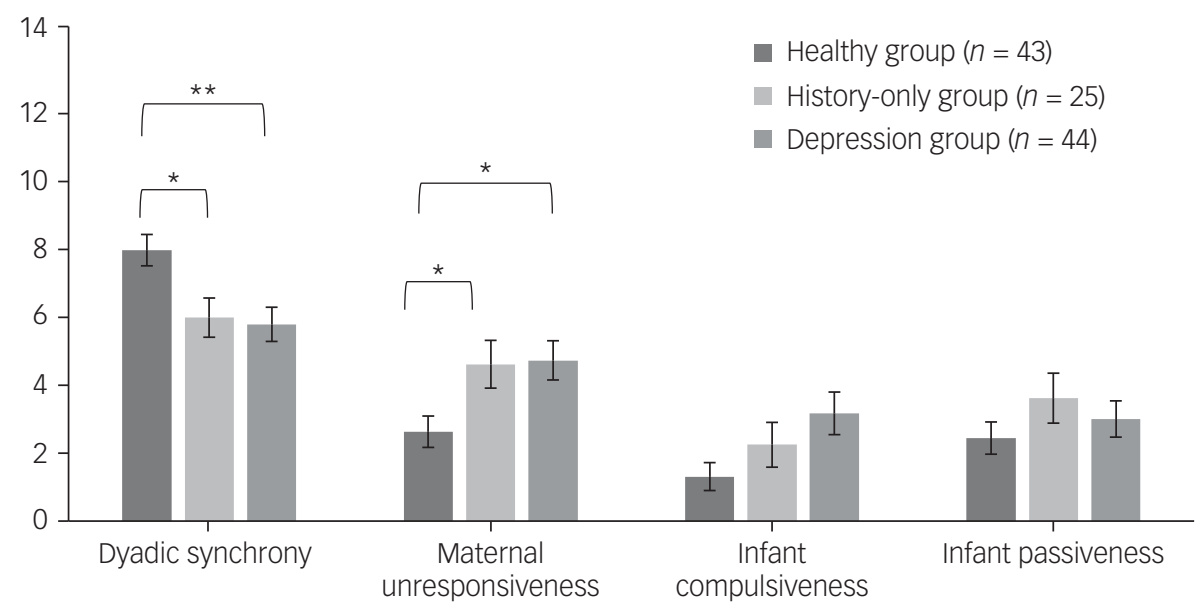

12 months postnatal

Fig. 2 Mean scores across patterns of behaviour on the Crittenden Child-Adult Relationship Experimental Index at 8 weeks and 12 months postnatal. ${ }^{*} P<0.05,{ }^{*} P<0.01$

dyadic synchrony and increased the variance explained to $13 \%$. However, the additions of PND and maternal group (depression/ history-only/healthy) did not further influence dyadic synchrony, and the total model explained $16 \%$ of the variance.

At 12 months, SES score was still significantly associated with dyadic synchrony and accounted for $20 \%$ of the variance; however, neither NBAS orientation, PND nor maternal group further influenced dyadic synchrony, and the total model explained $23 \%$ of the variance.

\section{Discussion}

This study examines the quality of mother-infant interactions, both early and late in the postnatal period, in healthy women, women with clinically significant depression in pregnancy and women with a lifetime history of depression but healthy pregnancies. We demonstrate that both depression groups have reduced dyadic synchrony at both 8 weeks and 12 months postnatal, as well as increased maternal unresponsiveness at 12 months postnatal. Contrary to previous literature, in univariate corelations, childhood maltreatment does not affect the mother-infant interaction in our sample, and PND may only have a marginal effect if occurring in the first 8 weeks postnatal. Of the clinical and sociodemographic factors in our sample that could explain these findings, maternal socioeconomic difficulties and dysregulation of neonatal orientation skills (ability to attend to auditory and visual stimuli, indicative of readiness for social interaction) appear to be the most likely contributory factors to reduced dyadic synchrony.

\section{Antenatal depression and mother-infant interaction}

Our findings in women with depression in pregnancy extend and confirm the previous evidence. Although no studies investigating antenatal depression have assessed dyadic synchrony, studies have found increased maternal unresponsiveness, even if measured just at a single assessment, late in the postnatal period; ${ }^{9,10}$ moreover, like ours, these studies measured both antenatal and postnatal depression, and found that the effects of antenatal symptoms are independent of, or stronger than, postnatal symptoms. As our study assesses dyadic synchrony in addition to maternal and infant domains of behaviour, both early and late in the postnatal period, our findings are both consistent with previous literature and novel. Interestingly, we also find that mothers in the depression group are more unresponsive at 12 months, but not at 8 weeks. This suggests that early on, women who are less sensitive are a mix 
between intrusive/controlling and withdrawn/unresponsive, but over time they become more withdrawn/unresponsive, perhaps because of prolonged depression or a new onset of PND after the first 8 weeks.

\section{Lifetime history of depression and mother-infant interaction}

Compared with healthy women, women with a lifetime history of depression but healthy pregnancies also have disrupted motherinfant interactions, with scores on the CARE-Index overlapping those of women in the depression group. To our knowledge, only one other study has explored this, which found that indeed parents (mothers and fathers) with histories of depression are more likely to show negative affect with their infants and less likely to show positive affect; again, this effect was independent of any postnatal depressive symptoms. ${ }^{11}$ The authors postulated that these findings might be a result of some of these mothers having had depression in pregnancy or early in the postpartum, which is something that they did not assess; however, our results show that the effects of a history of depression are present in the absence of either of these risk factors, as women in the history-only group in our study display minimal depressive symptoms at those time points. One possible neurobiological explanation for these persistent effects of past depression is changes in the levels of circulating oxytocin, a vital hormone for the formation of the mother-infant bond, ${ }^{12}$ as studies have found that women with depression (outside the perinatal period) have dysregulated oxytocin. ${ }^{13}$

\section{Variables predicting dyadic synchrony}

Our hierarchical regression models show that, at both 8 weeks and 12 months, the effect of group (depression/history-only/healthy) on dyadic synchrony is no longer significant upon consideration of the effects of maternal SES score and infant NBAS orientation score (at 8 weeks), or maternal SES score alone at 12 months. Indeed other studies have found that maternal sensitivity (a contributor to dyadic synchrony) can be predicted by socioeconomic factors, ${ }^{17}$ and that women of lower socioeconomic statuses may be less likely to partake in distal (visual and auditory) interactions. ${ }^{31}$ Also of note is that previous studies have found similar negative associations between NBAS scores and mother-infant interaction, ${ }^{19}$ such that reduced neonatal orientation is associated with decreased dyadic synchrony, but never in the context of maternal depression. Given that the orientation cluster of the NBAS assesses infants' abilities to engage in auditory and visual stimuli, such as interacting with the examiner by tracking their face or voice, this finding could indicate a route through which depression-related genetic vulnerability may translate to difficult infant temperament and thus a disrupted interaction, as suggested by studies showing that transmission of maternal depression into offspring behavioural alterations may be genetically driven. ${ }^{32}$ Moreover, it is also important to consider a transactional model of development, whereby the behaviour of each member of the dyad influences the response in the other, ${ }^{33}$ and thus parenting difficulties in mothers and temperament difficulties in infants may perpetuate each other.

Although it is impossible to make any inference on the factors causally responsible for the reduced quality of mother-infant interaction, our findings do suggest that maternal SES status and neonatal behaviour are part of a pathway that associates depression in mothers (both lifetime and specifically in pregnancy) with lesssynchronous mother-infant interaction. It is also important to highlight that women in the history-only group had SES scores that were significantly better than those of women in the depression group, whereas dyadic synchrony in both groups is equally disrupted. In fact, the higher SES score of women in the history-only group could represent the presence of protective factors that may have shielded them from becoming depressed during pregnancy (e.g. because they are more likely to be married/cohabiting, have financial security and/or have social support), but may not have been enough to preserve the quality of the interaction. This indicates that SES difficulties alone cannot causally explain our findings. Above all, when examining the effects that maternal psychopathology may have on offspring outcomes, it is important to consider that many women present with multiple vulnerabilities, and thus many factors, such as depression, socioeconomic vulnerability and infant behaviour, often overlap with each other, adding to the complexity of disentangling the effect that specific variables may have.

Our study also found that women in both the depression and history-only groups are more likely to be postnatally depressed than women in the healthy group, consistent with the notion that antenatal depression and a history of depression are both risk factors for PND. ${ }^{6}$ However, presence of PND itself does not contribute to the prediction of dyadic synchrony in the models, or in the univariate correlations when examined from birth through to 12 months, suggesting that women who experience PND alone may be protected in their interactions. Although many studies have looked at women who experience postnatal depression and have found less optimal interactions with their infants via reduced sensitivity and increased unresponsiveness/withdrawal, ${ }^{5}$ the majority of these studies have not taken into account antenatal symptoms and/ or a history of depression. The present study, and two others that looked at both antenatal and postnatal symptoms in the context of mother-infant interaction, found that antenatal depression is more predictive than PND in how the dyad will behave, ${ }^{9,10}$ possibly because antenatal depression may lead to disrupted foetal attachment and bonding early in the postpartum. In contrast, the presence of PND in itself does not detract from the mother-infant relationship if optimal bonding is present early on in the postnatal period, ${ }^{34}$ implying that the encouragement and support for the early relationship can buffer against maternal postnatal psychopathology. To date, other studies that have investigated the impact of perinatal depression on offspring outcomes have also found that antenatal depression is more predictive of outcomes, including offspring behavioural problems. ${ }^{35}$ Taken together, it is plausible that the differences found in studies only investigating postnatal symptoms may be at least partly due to antenatal or lifetime symptoms.

Furthermore, with regard to maternal anxiety, we found that antenatal anxiety was not associated with dyadic synchrony at either time point, suggesting that anxiety or stress in pregnancy do not hinder the development of the mother-infant interaction, consistent with previous literature. ${ }^{36}$ Anxiety at 8 weeks postnatal was correlated with dyadic synchrony at 12 months; however, given how closely anxiety was linked to PND, we expect that postnatal anxiety would not have contributed to dyadic synchrony, in the same way that PND did not in our hierarchical regression models.

Another surprising finding in our study is that although both women in the depression and history-only groups had significantly higher rates of childhood maltreatment, this was not associated with dyadic synchrony. Previous literature on maternal childhood maltreatment and mother-infant interaction has been mixed: some studies have found associations with disrupted mother-infant interactions, ${ }^{16}$ and others have not. ${ }^{37}$ Although a history of childhood maltreatment does increase the risk of perinatal depression, ${ }^{38}$ our study suggests that maternal history of childhood maltreatment may only affect subsequent mother-infant interactions because it is a risk factor for psychopathology, and in the absence of psychopathology (arguably, in resilient mothers), it does not in itself have an effect. 


\section{Limitations}

Our main limitation is that we may not have captured all of the important psychological factors relevant to the pathway from maternal depression to reduced quality of the mother-infant interaction, especially considering that the best of our regression models only explains $23 \%$ of the variance in dyadic synchrony. For example, much of the literature suggests that mothers are likely to mirror their own upbringing, ${ }^{39}$ but we have not evaluated mothers' attachment to their own mothers. Additionally, many studies find a link between foetal attachment status in pregnancy and mother-infant bonding quality in the postnatal period, ${ }^{7}$ but we did not measure foetal attachment. Another limitation is that we had some attrition throughout the study, although it was $<10 \%$ at each subsequent time point, and we found no differences between mothers who did and did not drop out. Finally, it is important to acknowledge that the sample size in our study was relatively small, and moreover, as the recruitment of the history-only group began at a later stage in the study, there were fewer women in this group than in the healthy and depression groups; although we believe that the difference in dyadic synchrony between the history-only group and the healthy group is still a robust finding given the magnitude of the effect, we recommend replication with a larger sample size.

\section{Clinical implications and conclusions}

The main clinical implication of our findings is that support for dyads at risk of interaction difficulties should begin antenatally, and also be targeted to well mothers with a history of depression, considering that history of depression is a major risk factor for becoming unwell in the perinatal period. At 8 weeks, already many of the dyads in the depression and history-only groups fall into the risk range on the CARE-Index, which, when used clinically, implies the need for parental treatment psychotherapy; ${ }^{30}$ and, furthermore, a significant proportion of these dyads continued to remain at risk at 12 months. Additionally, and perhaps surprisingly, even some healthy dyads, with neither past nor present psychopathology, fall into the inept range of the CARE-Index at 8 weeks postnatal, suggesting it may even be beneficial for all expectant mothers to receive support before giving birth. Moreover, given that the CARE-Index is used clinically within mother and baby units to assess dyads pre- and post-treatment, ${ }^{40}$ as well as in court cases to evaluate infant safety and risk, ${ }^{41}$ our study also contributes to the findings that it is a valid and robust clinical tool that can evaluate the quality and progression of the mother-infant relationship.

As pregnancy is a time when women are in contact with healthcare professionals, it is therefore an important goal to provide support in bonding with the foetus and future infant for all expectant mothers, not just for those who are unwell. This could be achieved by providing mothers with literature on examples of sensitive mothering behaviours, ways to engage with the foetus and with infants, behaviours to expect from an infant and developmental milestones. Additionally, educating mothers on sensitive behaviour, both with the foetus and the infant, could be incorporated into birthing classes and health visits. Finally, our findings highlight the importance of support from perinatal services for identified vulnerable women, especially across the postnatal period, and suggest that interventions proven to help the motherinfant interaction, such as video feedback ${ }^{42}$ and structured mother-baby activities, e.g., art and singing groups, ${ }^{43,44}$ should be more widely available. This way, we may be able to break the intergenerational transmission of psychopathology that begins with maternal depression (lifetime or in pregnancy) and may lead to increased mental health problems in offspring via, at least in part, a disrupted mother-infant interaction.
Rebecca H. Bind (D), Department of Psychological Medicine, Institute of Psychiatry, Psychology and Neuroscience, King's College London, UK; Alessandra Biaggi, Department of Psychological Medicine, Institute of Psychiatry, Psychology and Neuroscience, King's College London, UK; Aoife Bairead, Minds In Mind, Ireland; Andrea Du Preez, Department of Psychological Medicine, Institute of Psychiatry, Psychology and Neuroscience, King's College London, UK; and The Maurice Wohl Clinical Neuroscience Institute, Department of Basic and Clinical Neuroscience, Institute of Psychiatry, Psychology and Neuroscience, King's College London, UK;

Katie Hazelgrove, Department of Psychological Medicine, Institute of Psychiatry, Psychology and Neuroscience, King's College London, UK; Freddie Waites, Department of Psychological Medicine, Institute of Psychiatry, Psychology and Neuroscience, King's College London, UK; Susan Conroy, Department of Psychological Medicine, Institute of Psychiatry, Psychology and Neuroscience, King's College London, UK; Paola Dazzan, Department of Psychological Medicine, Institute of Psychiatry, Psychology and

Neuroscience, King's College London, UK; Sarah Osborne, Department of Psychological

Medicine, Institute of Psychiatry, Psychology and Neuroscience, King's College London, UK; Susan Pawlby, Department of Psychological Medicine, Institute of Psychiatry, Psychology and Neuroscience, King's College London, UK; Vaheshta Sethna, Sackler Institute for Translational Neurodevelopment, Department of Forensic \& Neurodevelopmental Sciences, Institute of Psychiatry, Psychology and Neuroscience, King's College London, UK; Carmine M. Pariante, Department of Psychological Medicine, Institute of Psychiatry, Psychology and Neuroscience, King's College London, UK

Correspondence: Rebecca H. Bind. Email: rebecca.bind@kcl.ac.uk

First received 16 Sep 2020, final revision 11 Mar 2021, accepted 8 Apr 2021

\section{Supplementary material}

Supplementary material is available online at https://doi.org/10.1192/bjo.2021.52.

\section{Data availability}

The data that support the findings of this study are available from the corresponding author, R.H.B., upon reasonable request.

\section{Acknowledgements}

We are grateful to the women and their infants who participated in the PRAM-D study, and to everyone on the study team who recruited, collected and analysed data.

\section{Author contributions}

R.H.B. contributed to the data curation, investigation, conceptualization, formal analysis, visualization, writing - original draft and writing - review \& editing. A. Biaggi contributed to the data curation, investigation, methodology, project administration and writing - review \& editing. A. Bairead contributed to the investigation and writing - review \& editing. A.D.P. contributed A. Bairead contributed to the investigation and writing - review \& editing. A.D.P. contributed
to the data curation, investigation, methodology, project administration and writing - review \& editing. K.H. contributed to the data curation, investigation, methodology, project administration and writing - review \& editing. F.W. contributed to the data curation and writing - review \& editing. S.C. contributed to the conceptualization, data curation, investigation, formal analysis, methodology, project administration and writing - review \& editing. P.D. contributed to the conceptualization, methodology and Writing - review \& editing. S.O. contributed to the conceptualization, methodology, investigation, data curation, project administration, writing - review \& editing and funding acquisition. S.P. contributed to the conceptualization, methodology, investigation, data curation, formal analysis, supervision and writing - review \& editing. V.S. contributed to the conceptualization, formal analysis, supervision and writing - review \& editing. C.M.P. contributed to the conceptualization, data curation, methodology, formal analysis, funding acquisition, supervision, writing - original draft and writing - review \& editing.

\section{Funding}

The work was supported by the following grants: UK National Institute for Health Research (NIHR) Senior Investigator Award (to C.M.P.); the NIHR Biomedical Research Centre at the South London and Maudsley NHS Foundation Trust and King's College London (to C.M.P., R.H.B. and S.O.): The Lullaby Trust (formerly known as the Foundation for the Study of Infant Deaths) (grant number 263) (to C.M.P., S.C. and S.O.); the Psychiatry Research Trust (to C.M.P. and S.O.) and the Brain and Behavior Research Foundation (to S.O.).

\section{Declaration of interest}

C.M.P. has received research funding from Johnson \& Johnson (2012-2018) and is funded by a Wellcome Trust strategy award to the Neuroimmunology of Mood Disorders and Alzheimer's Disease Consortium (number 104025), which is also funded by Janssen, GlaxoSmithKline, Lundbeck and Pfizer; the project is completely unrelated to this funding. P.D. received speaker's fees from Lundbeck. R.H.B., A. Biaggi, A. Bairead, A.D.P., K.H., F.W., S.C., S.O., S.P. and V.S. have nothing to declare.

ICMJE forms are in the supplementary material, available online at https://doi.org/10.1192/ bjo.2021.52. 


\section{References}

1 Luoma I, Tamminen T, Kaukonen P, Laippala P, Puura K, Salmelin R, et al. Longitudinal study of maternal depressive symptoms and child well-being. J Am Acad Child Adolesc Psychiatry 2001; 40(12): 1367-74.

2 Murray L, Fiori-Cowley A, Hooper R, Cooper P. The impact of postnatal depression and associated adversity on early mother-infant interactions and late infant outcome. Child Dev 1996; 67(5): 2512-26.

3 Field T. Maternal depression effects on infants and early interventions. Prev Med (Baltim) 1998; 27(2): 200-3.

4 Stein A, Gath DH, Bucher J, Bond A, Day A, Cooper PJ. The relationship between post-natal depression and mother-child interaction. Br J Psychiatry 1991; 158 (1): 46-52.

5 Field T. Postpartum depression effects on early interactions, parenting, and safety practices: a review. Infant Behav Dev 2010; 33(1): 1-6.

6 Biaggi A, Conroy S, Pawlby S, Pariante CM. Identifying the women at risk of antenatal anxiety and depression: a systematic review. J Affect Disord 2016; 191: $62-77$

7 Dubber S, Reck C, Müller M, Gawlik S. Postpartum bonding: the role of perinata depression, anxiety and maternal-fetal bonding during pregnancy. Arch Womens Ment Health 2015; 18(2): 187-95.

8 Rossen L, Hutchinson D, Wilson J, Burns L, Olsson CA, Allsop S, et al. Predictors of postnatal mother-infant bonding: the role of antenatal bonding, materna substance use and mental health. Arch Womens Ment Health 2016; 19(4): 609-22.

9 Flykt M, Kanninen K, Sinkkonen J, Punamäki R-L. Infant and child development maternal depression and dyadic interaction: the role of maternal attachment style. Inf Child Dev 2010; 19: 530-50.

10 Pearson RM, Melotti R, Heron J, Joinson C, Stein A, Ramchandani PG, et al. Disruption to the development of maternal responsiveness? The impact of prenatal depression on mother-infant interactions. Infant Behav Dev 2012: 35(4): $613-26$

11 Forbes $\mathrm{EE}$, Cohn JF, Allen NB, Lewinsohn PM. Infant affect during parent-infant interaction at 3 and 6 months: differences between mothers and fathers and influence of parent history of depression. Infancy 2004; 5(1): 61-84.

12 Galbally M, Lewis AJ, van IJzendoorn M, Permezel M. The role of oxytocin in mother-infant relations: a systematic review of human studies. Harv Rev Psychiatry 2011; 19(1): 1-14.

13 Cyranowski JM, Hofkens TL, Frank E, Seltman H, Cai H-M, Amico JA. Evidence of dysregulated peripheral oxytocin release among depressed women. Psychosom Med 2008; 70(9): 967-75.

14 Plant DT, Jones FW, Pariante CM, Pawlby S. Association between materna childhood trauma and offspring childhood psychopathology: mediation analysis from the ALSPAC cohort. Br J Psychiatry 2017; 211(3): 144-50.

15 Bolton HL, Hughes PM, Turton P, Sedgwick P. Incidence and demographic correlates of depressive symptoms during pregnancy in an inner London population. J Psychosom Obstet Gynecol 1998; 19(4): 202-9.

16 Vaillancourt K, Pawlby S, Fearon RMP. History of childhood abuse and motherinfant interaction: a systematic review of observational studies. Infant Ment Health J 2017; 38(2): 226-48.

17 Crittenden PM, Bonvillian JD. The relationship between maternal risk status and maternal sensitivity. Am J Orthopsychiatry 1984; 54(2): 250

18 Osborne S, Biaggi A, Chua TE, Du Preez A, Hazelgrove K, Nikkheslat N, et al. Antenata depression programs cortisol stress reactivity in offspring through increase maternal inflammation and cortisol in pregnancy: the Psychiatry Research an Motherhood - Depression (PRAM-D) study. Psychoneuroendocrinology 2018; 98 : 211-21.

19 Penman R, Meares R, Baker K, Milgrom-Friedman J. Synchrony in mother-infant interaction: a possible neurophysiological base. Br J Med Psychol 1983; 56(Pt 1): $1-7$

20 Pearson RM, Cooper RM, Penton-Voak IS, Lightman SL, Evans J. Depressive symptoms in early pregnancy disrupt attentional processing of infant emotion. Psychol Med 2010; 40(4): 621-31.

21 Evans GW, Li D, Whipple SS. Cumulative risk and child development. Psychol Bull 2013; 139(6): 1342.
22 Bifulco A, Bernazzani O, Moran PM, Jacobs C. The Childhood Experience of Care and Abuse Questionnaire (CECA.Q): validation in a community series. $\mathrm{Br} J \mathrm{Clin}$ Psychol 2005; 44(4): 563-81.

23 Plant DT, Pariante CM, Sharp D, Pawlby S. Maternal depression during pregnancy and offspring depression in adulthood: role of child maltreatment. $\mathrm{Br} J$ Psychiatry 2015; 207(3): 213-20.

24 First MB, Spitzer RL, Gibbon M, Williams JBW. Structured Clinical Interview for DSM-IV Axis I Disorders-Patient Edition (SCID-I/P, Version 2.0). New York State Psychiatric Institute, 1995.

25 Beck AT, Ward C, Mendelson M, Mock J, Erbaugh J. Beck Depression Inventory (BDI). Arch Gen Psychiatry 1961; 4(6): 561-71.

26 Spielberger CD. Manual for the State-Trait Anxiety Inventory STAI (Form Y) ("Self-Evaluation Questionnaire"). American Psychological Association, 1983 (https://psycnet.apa.org/record/9999-06496-000?doi=1)

27 Brazelton TB, Nugent JK. Neonatal Behavioral Assessment Scale (NBAS). MacKeith Press, 1995

28 Greene JG, Fox NA, Lewis M. The relationship between neonatal characteristics and three-month mother-infant interaction in high-risk infants. Child Dev 1983; 54(5): 1286-96.

29 Crittenden PM. CARE-Index Infants (Birth-15 Months) Coding Manual. Family Relations Institute, 2004.

30 Crittenden PM. CARE-Index Manual. Family Relations Institute, 2003.

31 Field T, Pawlby S. Early face-to-face interactions of British and American working-and middle-class mother-infant dyads. Child Dev 1980; 51: 250-3.

32 O'Donnell KJ, Meaney MJ. Fetal origins of mental health: the developmental origins of health and disease hypothesis. Am J Psychiatry 2017; 174(4): 319-28.

33 Coulacoglou C, Saklofske DH. Chapter 8 - The assessment of family, parenting, and child outcomes. In Psychometrics and Psychological Assessment: Principles and Applications: 187-222. Academic Press, 2017.

34 Rossen L, Mattick RP, Wilson J, Clare PJ, Burns L, Allsop S, et al. Mother-infant bonding and emotional availability at 12-months of age: the role of early postnatal bonding, maternal substance use and mental health. Matern Child Health J 2019; 23(12): 1686-98.

35 Waters CS, Hay DF, Simmonds JR, van Goozen SHM. Antenatal depression and children's developmental outcomes: potential mechanisms and treatment options. Eur Child Adolesc Psychiatry 2014; 23(10): 957-71.

36 Nath S, Pearson RM, Moran P, Pawlby S, Molyneaux E, Challacombe FL, et al The association between prenatal maternal anxiety disorders and postpartum perceived and observed mother-infant relationship quality. J Anxiety Disord 2019; 68: 102148

37 Leon K, Jacobvitz D, Hazen N. Maternal resolution of loss and abuse: associations with adjustment to the transition to parenthood. Artic Infant Ment Heal J 2004; 25(2): 130-48.

38 Plant DT, Barker ED, Waters CS, Pawlby S, Pariante CM. Intergenerational transmission of maltreatment and psychopathology: the role of antenatal depression. Psychol Med 2013; 43(3): 519-28.

39 Van lizendoorn $\mathrm{MH}$. Intergenerational transmission of parenting: a review of studies in nonclinical populations. Dev Rev 1992; 12(1): 76-99.

40 Stephenson LA, Macdonald AJD, Seneviratne G, Waites F, Pawlby S. Mother and baby units matter: improved outcomes for both. BJPsych Open 2018; 4(3): 119-25.

41 Ostler T. Assessing parenting risk within the context of severe and persistent mental illness: validating an observational measure for families with child protective service involvement. Infant Ment Health J 2010; 31(5): 467-85.

42 Kenny M, Conroy S, Pariante CM, Seneviratne G, Pawlby S. Mother-infant interaction in mother and baby unit patients: before and after treatment. J Psychiatr Res 2013; 47(9): 1192-8.

43 Fancourt $D$, Perkins R.. Effect of singing interventions on symptoms of postnatal depression: three-arm randomised controlled trial. $\mathrm{Br} J$ Psychiatry 2018; 212(2): 119-21

44 Carolina E, Daisy F, Paola D, Ray CK, Nick S, Anthony W, et al. Scaling-up HealthArts Programmes: the largest study in the world bringing arts-based mental health interventions into a national health service. BJPsych Bull 2021; 45(1): 32-9.

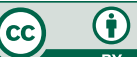

